\title{
An Astrocytic Influence on Impaired Tonic Inhibition in Hippocampal CA1 Pyramidal Neurons in a Mouse Model of Rett Syndrome
}

\author{
${ }^{\circledR}$ Qiping Dong, ${ }^{1}{ }^{\circledR}$ Jason Kim, ${ }^{1}$ Linh Nguyen, ${ }^{1}{ }^{\circledR}$ Qian Bu, ${ }^{1}$ and ${ }^{\circledR}$ Qiang Chang ${ }^{1,2,3}$ \\ ${ }^{1}$ Waisman Center, University of Wisconsin-Madison, Madison, Wisconsin 53705, ${ }^{2}$ Department of Medical Genetics, University of Wisconsin- \\ Madison, Madison, Wisconsin 53705, and ${ }^{3}$ Department of Neurology, University of Wisconsin-Madison, Madison, Wisconsin 53705
}

Rett syndrome (RTT) is a severe neurodevelopmental disease caused by mutations in the methyl-CpG binding protein 2 (MECP2) gene. Although altered interneuron development and function are clearly demonstrated in RTT mice, a particular mode of inhibition, tonic inhibition, has not been carefully examined. We report here that tonic inhibition is significantly reduced in pyramidal neurons in the CA1 region of the hippocampus in mice where Mecp2 is deleted either in all cells or specifically in astrocytes. Since no change is detected in the level of GABA receptors, such a reduction in tonic inhibition is likely a result of decreased ambient GABA level in the extracellular space. Consistent with this explanation, we observed increased expression of a GABA transporter, GABA transporter 3 (GAT3), in the hippocampus of the Mecp2 KO mice, as well as a corresponding increase of GAT3 current in hippocampal astrocytes. These phenotypes are relevant to RTT because pharmacological blockage of GAT3 can normalize tonic inhibition and intrinsic excitability in CA1 pyramidal neurons, and rescue the phenotype of increased network excitability in acute hippocampal slices from the Mecp2 KO mice. Finally, chronic administration of a GAT3 antagonist improved a composite symptom score and extended lifespan in the Mecp2 KO mice. Only male mice were used in this study. These results not only advance our understanding of RTT etiology by defining a new neuronal phenotype and revealing how it can be influenced by astrocytic alterations, but also reveal potential targets for intervention.

Key words: astrocyte; GABA transporter; MeCP2; mouse model; Rett syndrome; tonic inhibition

Significance Statement

Our study reports a novel phenotype of reduced tonic inhibition in hippocampal CA1 pyramidal neurons in the Rett syndrome mice, reveal a potential mechanism of increased GABA transporter expression/activity in the neighboring astrocytes, describe a disease-relevant consequence in hyperexcitability, and provide preliminary evidence that targeting this phenotype may slow down disease progression in Rett syndrome mice. These results help our understanding of the disease etiology and identify a new therapeutic target for treating Rett syndrome.

\section{Introduction}

Rett syndrome (RTT) is caused by mutations in the X-linked MECP2 gene encoding the methyl CpG-binding protein 2 (MECP2) (Amir et al., 1999), which is widely expressed and plays a vital role in the epigenetic regulation of gene expression. RTT patients often experience normal development during the first 6-

Received Dec. 25, 2019; revised May 13, 2020; accepted June 10, 2020.

Author contributions: Q.D. and Q.C. designed research; Q.D., J.K., L.N., and Q.B. performed research; Q.D. analyzed data; $Q . D$. and Q.C. edited the paper; Q.C. wrote the first draft of the paper; Q.C. wrote the paper.

This work was supported in part by National Institutes of Health Grants R01HD064743 and R56NS100024 to Q.C., and National Institutes of Health Grant U54HD090256 to the Waisman Center.

The authors declare no competing financial interests.

Correspondence should be addressed to Qiang Chang at qchang@waisman.wisc.edu.

https://doi.org/10.1523/JNEUROSCI.3042-19.2020

Copyright $\odot 2020$ the authors
18 months, which is followed by a rapid developmental regression. Mecp2 KO (Mecp $\left.2^{-/ y}\right)$ mice have been created to model the human disease (Chen et al., 2001; Guy et al., 2001). Most of the male Mecp2 KO mice develop RTT-like phenotypes and die within the first few months of life, while the female Mecp2 heterozygous $\left(\operatorname{Mecp}^{+/-}\right)$mice become symptomatic at a much later time point.

GABAergic signaling plays a critical role in the development and function of the brain. Alterations in GABAergic interneuron development and inhibitory transmission are involved in the development of a number of neurologic disorders, including RTT (Nelson and Valakh, 2015; Lee et al., 2017). Deletion of Mecp2 solely in GABAergic neurons is sufficient to recapitulate most of the RTT-like phenotypes of the Mecp2 KO mouse, including ataxia, stereotyped behaviors, seizures, breathing abnormalities, and premature death (Chao et al., 2010; He et al., 2014; Ito- 
Ishida et al., 2015). Furthermore, restoration of MeCP2 expression in GABAergic neurons rescues many disease features in a mouse model of RTT (Ure et al., 2016). Finally, precocious GABAergic interneuron development (Krishnan et al., 2015, 2017) has been suggested to underlie regression like phenotypes in the visual system (Noutel et al., 2011; Durand et al., 2012) in the Mecp2 $\mathrm{KO}$ mice.

There are two modes of inhibitions in the CNS: phasic inhibition and tonic inhibition (Farrant and Nusser, 2005). In contrast to phasic inhibitory currents mediated by postsynaptic $\mathrm{GABA}_{\mathrm{A}}$ receptors $\left(\mathrm{GABA}_{\mathrm{A}} \mathrm{Rs}\right)$, tonic inhibitory currents are mediated by ambient GABA and activation of the extrasynaptic $\mathrm{GABA}_{\mathrm{A}}$ Rs. Tonic inhibition has been observed in different brain regions and different types of neurons (Bai et al., 2001; Nusser and Mody, 2002; Porcello et al., 2003; Semyanov et al., 2003; Caraiscos et al., 2004; Cope et al., 2005). The distinct subunit composition and the cellular distribution endow the extrasynaptic $\mathrm{GABA}_{\mathrm{A}} \mathrm{Rs}$ many properties different from their synaptic counterparts, such as high affinity for GABA, slow kinetics, and less extensive desensitization (Farrant and Nusser, 2005; Brickley and Mody, 2012). Many lines of evidence support the important role of tonic inhibition in maintaining physiological network activity, including the observation of abnormal tonic inhibition in different neurologic disease models (Brickley and Mody, 2012). Although some recent studies have described dysregulated tonic inhibition in some autism spectrum disorders (Curia et al., 2009; Egawa et al., 2012; Bridi et al., 2017), the underlying mechanisms are not well understood. In the context of the demonstrated role of altered GABAergic interneuron development and function in RTT and the lack of understanding of the role of tonic inhibition in RTT, we studied the tonic inhibitory currents in hippocampal CA1 pyramidal neurons in a mouse model of RTT. Here, we report an impairment of tonic inhibition in symptomatic Mecp2 KO mice, which can be attributed to enhanced GABA transporter (GAT) activity in astrocytes and contribute to the hyperexcitability of CA1 pyramidal neurons. Our findings provide new insights into the etiology of RTT and identify potential new targets for future therapeutic development.

\section{Materials and Methods}

Animals. The Mecp2 $\mathrm{KO}$ mice used in this study are the Jaenisch strain (Mecp2 $2^{\text {tm1.1jae }}$ ) (Chen et al., 2001). Mice were housed in a facility with $12 \mathrm{~h}$ light/12 h dark cycle. Experimental protocols were approved by the Institutional Animal Care and Use Committee of the University of Wisconsin-Madison and conform to the guidelines of the Research Animal Resources and Compliance at the University of WisconsinMadison and the National Institutes of Health's Guide for the care and use of laboratory animals.

Immunofluorescence. For immunohistological evaluation on brain sections, mice were killed by barbiturate overdose and perfused transcardially with PBS, followed by $4 \%$ PFA (in PBS). Brains were removed and postfixed overnight. Then the brains were cryoprotected in buffered $30 \%$ sucrose (w/v) for at least $2 \mathrm{~d}$. The $40 \mu \mathrm{m}$ coronal frozen sections were prepared using a cryostat (Microm, HM 505N). Immunostaining was performed as previously described (Dong et al., 2018). Briefly, sections were permeabilized with $1 \%$ Triton X-100 (Sigma Millipore, in PBS) for 30 min, blocked with 3\% normal donkey serum and $0.25 \%$ Triton X-100 in PBS for $90 \mathrm{~min}$ at room temperature, and then incubated with primary antibodies overnight at $4^{\circ} \mathrm{C}$. Then the sections were incubated with the corresponding secondary antibodies for $2 \mathrm{~h}$ at room temperature and washed 5 times with PBS at room temperature. Primary antibody dilutions were as follows: anti-GABA (A2052, Sigma Millipore, 1:500), anti-GABA transporter 3 (GAT3) (AB1574, Millipore, 1:500). Secondary antibody dilutions were as follows: AlexaFluor-568 donkey-anti-rabbit antibody (Invitrogen, A10042, 1:500), AlexaFluor-488 donkey-anti-mouse antibody (Invitrogen, A21202, 1:500), and AlexaFluor-647 donkey-antimouse antibody. DAPI was used at $3 \mathrm{~nm}$ for counterstaining. Images were taken using a Nikon Al confocal microscope. Imaging fields were randomly selected in each coronal hippocampal section. Two or three sections were analyzed from each mouse. Three mice were included in each genotype. The mean fluorescence intensity of GABA or GAT3 in CA1 stratum radiatum was then quantified using ImageJ by investigators blind to the mouse genotype information. The $\mathrm{GABA}^{+}$cell number were counted manually using the Cell Counter plugin from ImageJ.

Western blot analysis. Hippocampi from 8- to 10-week-old WT and Mecp2 KO mice were collected into an Eppendorf tube and directly lysed by adding $1 \times$ LDS buffer (Invitrogen). Samples were sonicated to facilitate cell lysis and boiled for $5 \mathrm{~min}$ before loading into 10\% SDS-PAGE gels. Proteins were transferred onto nitrocellulose membrane (Whatman) using a semidry transfer system from Bio-Rad. The membrane was first blocked with 5\% milk solution for $1 \mathrm{~h}$ and then incubated with anti-GAT3 antibody (1:1000, AB1574, Millipore) diluted in $3 \%$ BSA solution at $4^{\circ} \mathrm{C}$ overnight. After incubating with infrared dye-conjugated secondary antibody (1:10,000, Thermo Fisher Scientific) for $1 \mathrm{~h}$ at room temperature, membrane was scanned in an Odyssey infrared imaging system.

Acute brain slice preparation. Male WT or Mecp2 KO mice were killed, and the brains were isolated. Using a microtome (VT1000S, Leica Microsystems), coronal brain slices $(400 \mu \mathrm{m})$ were prepared in ice-cold modified aCSF (in mM as follows: $124 \mathrm{NaCl}, 2.5 \mathrm{KCl}, 0.5 \mathrm{CaCl}_{2}, 5$ $\mathrm{MgCl}_{2}, 1.25 \mathrm{NaH}_{2} \mathrm{PO}_{4}, 26 \mathrm{NaHCO}_{3}$, and 15 glucose) saturated with $95 \%$ $\mathrm{O}_{2} / 5 \% \mathrm{CO}_{2}$. Then the slices were incubated in normal aCSF (in $\mathrm{mm}$ as follows: $124 \mathrm{NaCl}, 2.5 \mathrm{KCl}, 2.5 \mathrm{CaCl}_{2}, 1.2 \mathrm{MgCl}_{2}, 1.25 \mathrm{NaH}_{2} \mathrm{PO}_{4}, 26$ $\mathrm{NaHCO}_{3}$, and 15 glucose) at room temperature for at least $1 \mathrm{~h}$.

Electrophysiological recording. Slices were transferred to a recording chamber (Warner Instruments) perfused with $95 \% \mathrm{O}_{2} / 5 \% \mathrm{CO}_{2}$ saturated aCSF. Whole-cell patch-clamp recordings were made using a Multipatch 700B preamplifier, digitized with a Digidata $1440 \mathrm{~A}$, and acquired using pCLAMP 10 (Molecular Devices). The pipette solution contained the following (in $\mathrm{mm}$ ): 115 Cs-methanesulfonate, 25 TEA, 10 HEPES, 10 QX-314, $4 \mathrm{NaCl}, 4 \mathrm{Mg}$-ATP, 1 EGTA, 0.3 Li-GTP, and 10 phosphocreatine, $\mathrm{pH}$ 7.2. To record the inhibitory $\mathrm{GABA}_{\mathrm{A}} \mathrm{R}$ current, hippocampal CA1 neurons were clamped at $0 \mathrm{mV}$.

Tonic $\mathrm{GABA}_{\mathrm{A}} \mathrm{R}$-mediated current $\left(I_{\text {tonic }}\right)$ was determined as the shift of baseline holding currents after switching the superfusion solution from normal aCSF to aCSF containing $\mathrm{GABA}_{\mathrm{A}} \mathrm{R}$ antagonist bicuculline $(20 \mu \mathrm{M})$. All-points histograms were generated from recording currents for the control and bicuculline period, respectively. The histograms were then fitted with a Gaussian distribution, and only one side of the histograms was used to avoid IPSC contamination (Bright and Smart, 2013). The change in the peaks of the Gaussian curves was used as the magnitude for $I_{\text {tonic }}$ (see Fig. $1 A$ ).

To record GAT-mediated current, astrocytes from hippocampal CA1 stratum radiatum were recognized by their distinct morphology and recorded. Recording pipettes (5-8 $\mathrm{M} \Omega$ ) were filled with a solution containing the following (in $\mathrm{mM}$ ): $125 \mathrm{Cs}$-methanesulfonate, $5 \mathrm{KCl}, 10$ HEPES, $4 \mathrm{NaCl}, 4 \mathrm{Mg}$-ATP, 10 EGTA, $0.3 \mathrm{Li}-\mathrm{GTP}$, and 10 phosphocreatine, $\mathrm{pH} 7.2$

GAT currents were induced by a fast focal application of GABA (1 mM)-containing aCSF with a pipette near the recorded astrocyte. TTX $(1 \mu \mathrm{M})$ and bicuculline $(20 \mu \mathrm{M})$ were added to the perfusion aCSF. GAT3-selective currents were isolated with selective antagonists.

In the current-clamp recording and in vitro epilepsy model, male mice at postnatal 5-8 weeks of age were used. The pipette solution contained the following (in $\mathrm{mm}$ ): $140 \mathrm{~K}$-gluconate, $7.5 \mathrm{KCl}, 10 \mathrm{HEPES}-\mathrm{K}$, 0.5 EGTA-K, $4 \mathrm{Mg}$-ATP, and $0.3 \mathrm{Li}-\mathrm{GTP}$. To evaluate the neuronal intrinsic excitability, currents were injected into pyramidal neurons, and the firing rate was quantified. For the in vitro epilepsy model, the perfused aCSF contained $5 \mathrm{~mm} \mathrm{KCl}$. Epileptiform activity was induced by switching perfused solution to aCSF containing $0 \mathrm{Mg}^{2+}$. Epileptiform events were detected with Clampfit and verified visually. The currents caused by unclamped action potentials have been truncated.

Tamoxifen injection. GFAP-CreERT;Mecp $2^{\text {flox/y }}$ mice and their littermate control Mecp $2^{\text {flox/y }}$ mice received daily intraperitoneal injection of 
tamoxifen $(80 \mathrm{mg} / \mathrm{kg})$ for $7 \mathrm{~d}$ when they were $\sim 6$ weeks old. Mice were killed and used for experiments 2-3 weeks after the injection. To test the specificity of the tamoxifen-induced Cre expression, Ail4;GFAPCreERT mice were used and different cell markers (NG2, NeuN, and GFAP) were stained.

$\beta$-alanine treatment. Male Mecp2 KO mice were randomly separated into vehicle $(0.9 \%$ saline $10 \mathrm{ml} / \mathrm{kg}$, i.p.) and $\beta$-alanine $(31 \mathrm{mg} / 10 \mathrm{ml} / \mathrm{kg}$, i.p.) groups. Mice were administered with vehicle or $\beta$-alanine starting at 4 weeks of age. The body weight and the disease severity scores of mice were monitored weekly. The score scheme was described previously (Guy et al., 2007). In brief, the symptoms of inertia, gait, hindlimb clasping, tremor, irregular breathing, and poor general condition of mice were evaluated and scored from 0 (absent), 1 (present), to 2 (severe) for each symptom. Mice losing $20 \%$ body weight were killed on humane grounds.

Statistics analysis. All data were analyzed using SigmaPlot 13.0 (Systat Software). Student's $t$ tests or Mann-Whitney $U$ tests were used to make comparisons between two groups unless indicated otherwise. Oneway ANOVA followed by post hoc HolmSidak test was performed when there are three or more groups. Log-rank (MantelCox) test was used in the survival analysis. Average data are shown as the mean \pm SEM. $p$ values $<0.05$ were considered statistically significant. No statistical methods were used to predetermine sample sizes.

\section{Results}

Reduced tonic GABA current in hippocampal CA1 pyramidal neurons from Mecp2 KO mice

To compare GABA-mediated tonic currents between wild type (WT) littermates and Mecp2 $\mathrm{KO}$ mice, whole-cell voltage-clamp recordings were conducted in hippocampal CA1 pyramidal neurons in acute brain slices prepared from male mice at postnatal day $(\mathrm{P})$ 56-70. Tonic GABA $\mathrm{A}$ R-mediated current $\left(I_{\text {tonic }}\right)$ was induced by bath application of $20 \mu \mathrm{M}$ bicuculline. Bicuculline blocked the sIPSCs and reduced the holding current, thereby revealing a tonically active GABA current (Fig. 1A). Comparing WT and Mecp2 KO neurons, $I_{\text {tonic }}$ decreased by $35.5 \%$ in Mecp2 KO mice $(19.83 \pm$ $2.114 \mathrm{pA}, N=12$ cells from 4 mice) compared with WT mice (30.75 $\pm 2.219 \mathrm{pA}, N=12$ cells from 4 mice; $t_{(22)}=3.56, p=0.002$; Fig. $1 B)$. To find out whether reduced tonic inhibition is associated with certain stage of disease progression, we recorded from hippocampal CA1 pyramidal neuron in acute brain slices prepared from juvenile (P21-P28) Mecp2 KO mice with no overt symptoms. At this younger age, no significant difference in tonic inhibition was detected between the Mecp2 KO mice and their WT littermates (WT: $33.86 \pm 1.908 \mathrm{pA}, N=7$ cells from 3 mice; Mecp2 KO: $32.14 \pm 2.97 \mathrm{pA}, N=7$ cells from 3 mice; $t_{(12)}=0.49, p=0.64$; Fig. 1C). This result indicated that the tonic inhibition phenotype in the Mecp2 $\mathrm{KO}$ mice is age-dependent and only manifests in the symptomatic mice.
Reduced ambient GABA level, but not reduced GABA receptor level, likely underlies the decreased tonic GABA currents

The impaired tonic inhibition in Mecp2 $\mathrm{KO}$ mice could be a result of either decreased GABA receptor (GABAR) level or reduced ambient GABA level. To distinguish between the two scenarios, we examined the GABA ( $5 \mu \mathrm{M}$, puff)-evoked currents with whole-cell patch-clamp recording in hippocampal CA1 neurons from WT and symptomatic Mecp2 KO mice. No difference was detected in the amplitudes of the GABA-evoked currents between the two genotypes (WT: $1.131 \pm 0.096 \mathrm{nA}, N=8$ cells from 3 mice; Mecp2 KO: $1.227 \pm 0.098 \mathrm{nA}, N=8$ from 3 mice; Mann-Whitney Rank Sum Test $U=23.0, p=0.38$; Fig. $2 A)$. This result suggests no alteration in the level of functional GABARs in hippocampal CA1 pyramidal neurons in the Mecp2 $\mathrm{KO}$ mice. Since it is reported that tonic inhibition in mouse hippocampal CA1 pyramidal neurons is mediated by $\alpha 5$ subunit-containing $\mathrm{GABA}_{\mathrm{A}}$ Rs (Caraiscos et al., 2004), we further investigated the contribution of $\alpha 5$ subunit-containing $\mathrm{GABA}_{\mathrm{A}} \mathrm{Rs}$ by using $\mathrm{GABA}_{\mathrm{A}} \mathrm{R}$ agonist isoguvacine (Iso, $20 \mu \mathrm{M}$ ) and $\alpha 5$ subunit-selective reverse agonist L-655708 to isolate the L-655708-sensitive current (Fig. 2B). No significant difference was detected in the 

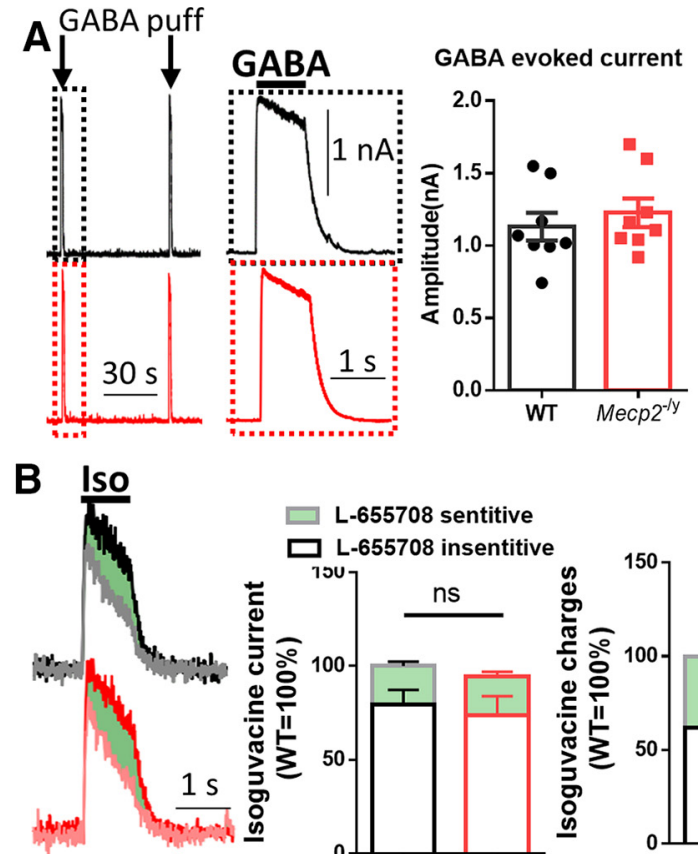

C
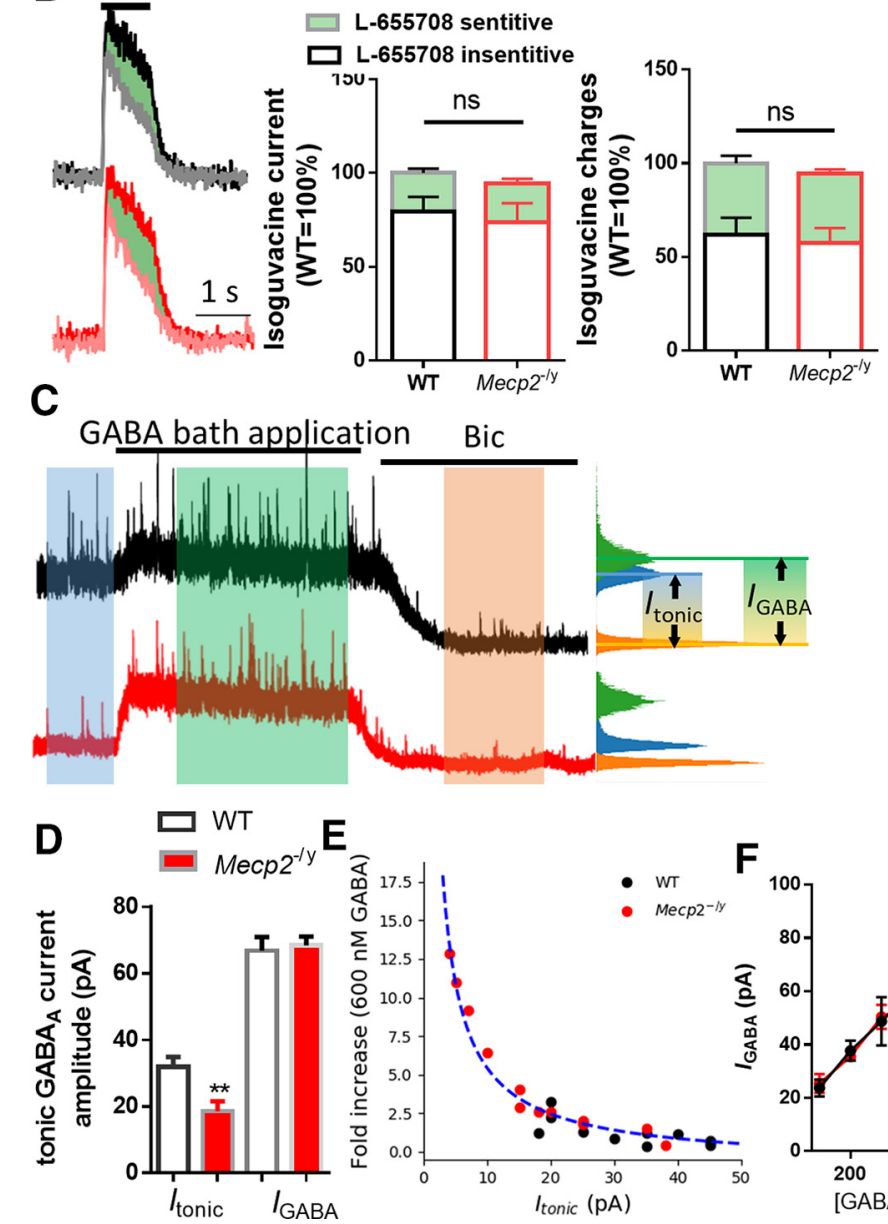

Bic
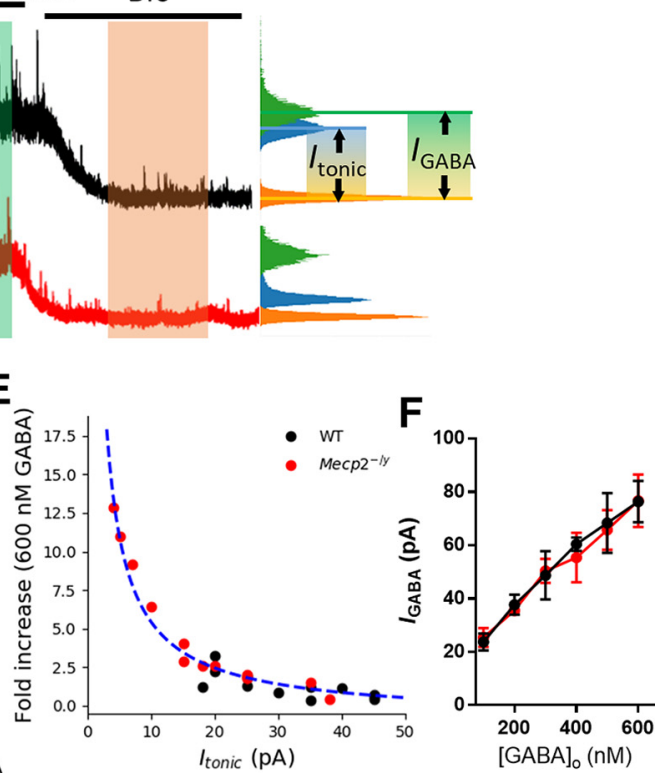

Figure 2. Reduced ambient GABA level underlies the decreased tonic GABA currents. $\boldsymbol{A}$, Left, Representative recording traces showing currents evoked by local puffing application of GABA from WT (black) and Mecp2 KO (red) mice. Inset, Magnified view of the boxed event. Calibration: $30 \mathrm{~s}(x$ axis), $1 \mathrm{~s}$ ( $x$ axis, inset), $1 \mathrm{nA}$ ( $y$ axis). Right, Quantification of the amplitudes of GABAevoked currents from WT and Mecp2 KO (Mecp2 ${ }^{-1 y}$ ) mice. $\boldsymbol{B}$, Left, Representative recording traces showing currents evoked by local puffing application of GABA receptor-selective agonist isoguvacine (Iso) from WT (black) and Mecp2 KO (red) mice. Gray and pink traces represent the Iso current in the presence of L-655708, a selective $\alpha 5-G A B A_{A} R$ reverse agonist. Middle, Quantification of Iso current amplitudes and the part of L-655708-sensitive current (green). Right, Quantification of Iso current charges and the part of L-655708-sensitive current (green). C, Representative traces showing the effects of $600 \mathrm{~nm}$ GABA bath application on holding currents. D, Quantification of amplitudes of tonic holding currents induced by endogenous (Itonic $)$ and exogenous $\left(I_{\text {GABA }}\right)$ GABA of WT and Mecp2 KO (Mecp2 $2^{-1 y}$ ) mice. $I_{\text {tonic }}$ and $I_{\text {GABA }}$ are indicated in $\boldsymbol{C}$. $\boldsymbol{E}$, The fold increase of $I_{\text {GABA }}$ against $I_{\text {tonic }}$ is plotted for each cell recorded from WT (black dots) and Mecp2 KO (red dots) mice. The fitted curve (blue dashed line) is calculated from all cells with a Hill equation. $\boldsymbol{F}, I_{\text {GABA }}$ induced by exogenous GABA of different concentrations from WT (black) and Mecp2 KO (Mecp2 ${ }^{-/ y}$, red) mice. ${ }^{* *} p<0.01$ versus WT in $\boldsymbol{E}$. ns, not significant.

amplitude of Iso current between the WT and Mecp2 KO mice (WT: $100 \pm 9.85 \%, N=6$ cells from 3 mice; Mecp2 KO: $94.40 \pm 12.60 \%, N=6$ cells from 3 mice; $\left.t_{(10)}=0.35, p=0.73\right)$. The amplitude of L-655708-sensitive currents was also comparable between WT $(20.50 \pm 2.29 \%, N=6$ cells from 3 mice) and Mecp2 $\mathrm{KO}(20.67 \pm$ $2.59 \%, N=6$ cells from 3 mice) groups $\left(t_{(10)}=-0.04, p=0.96\right)$. Finally, the total charges mediated by the currents were also quantified, and no significant difference was found between WT (Iso current: $100 \pm 5.98 \%$; L-655708-sensitive current: $37.96 \pm 3.98 \% ; N=6$ cells from 3 mice) and Mecp2 KO (Iso current: 94.41 $\pm 7.71 \% ; t_{(10)}=0.58, p=0.58 ; \mathrm{L}-$ 655708-sensitive currents: $36.77 \pm$ $2.39 \% ; t_{(10)}=0.26, p=0.80$ ) groups.

Previously, El-Khoury et al. (2014) compared the ambient GABA concentration between the WT and Mecp2 KO mice and reported that the GABA level is spatially and developmentally regulated. More specifically, the symptomatic Mecp2 KO mice exhibit reduced extracellular GABA levels in the hippocampus. Their result is consistent with our electrophysiological recording results, which could explain the reduced tonic inhibition observed in these mice. To further validate this hypothesis, we superfused acute brain slices with 600 nм GABA, which is higher than the estimated average extrasynaptic GABA concentration $160 \mathrm{~nm}$ (Santhakumar et al., 2006). We found that such GABA superfusion abolished the difference in tonic inhibition between the WT and Mecp2 KO mice (WT: $66.79 \pm$ $4.123 \mathrm{pA}, \mathrm{N}=12$ cells from 3 mice; Mecp2 KO: $68.42 \pm 2.63 \mathrm{pA}, N=13$ cells from 3 mice; $t_{(23)}=-0.338, p=0.74$; Fig. $2 C, D)$. The fold increase of $I_{\mathrm{GABA}}$ against $I_{\text {tonic }}$ from both genotypes showed an inverse correlation. The plot was well fitted with a Hill equation (Fig. 2E). $I_{\mathrm{GABA}}$ induced by different concentration (100$600 \mathrm{~nm}$ ) of GABA was also recorded. No significant difference was detected in the amplitude of current evoked by any concentration of GABA between WT and Mecp2 KO mice (Fig. 2F). Together, these results suggest that the decreased tonic inhibition observed in the Mecp2 $\mathrm{KO}$ mice is likely a result of lower ambient GABA level in the extracellular space rather than decreased GABA receptor density.

\section{Enhanced GAT activity in astrocytes from the Mecp2 $\mathrm{KO}$ mice}

The extracellular GABA level is regulated by both release and uptake. We first compared the synaptic release of GABA between the WT and Mecp2 KO mice. Although the Mecp2 $\mathrm{KO}$ mice showed slightly lower mIPSC amplitude than the WT mice (Fig. 3A; no statistical significance), they showed a similar level of sIPSC amplitude as 
A
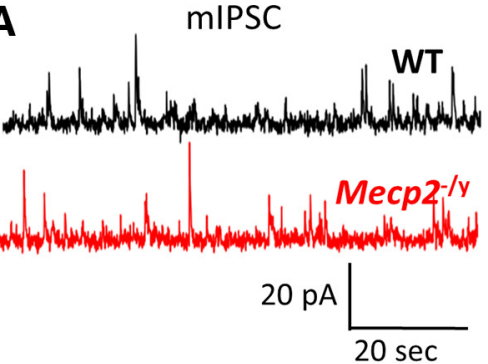

B

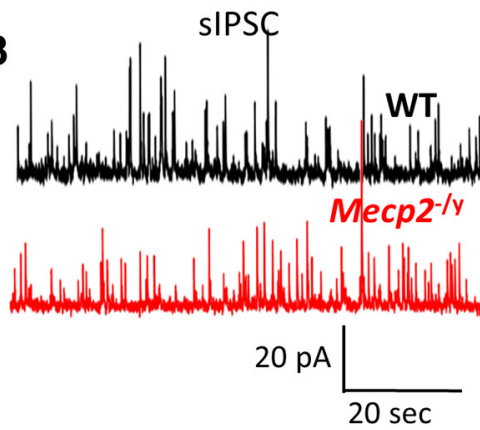

C

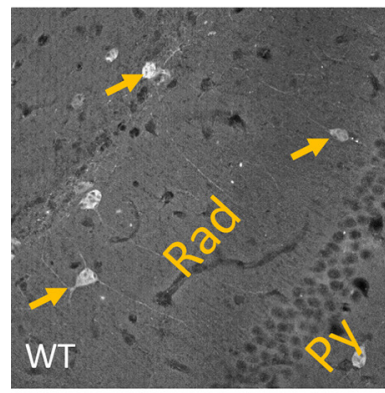

E

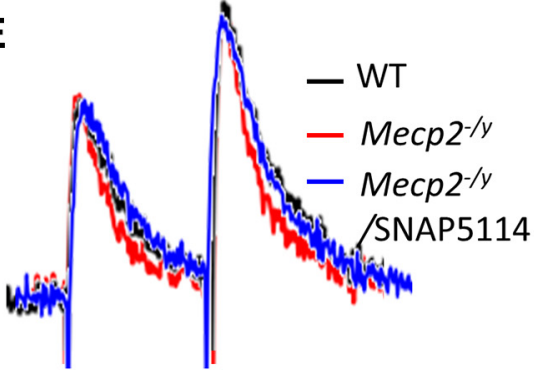

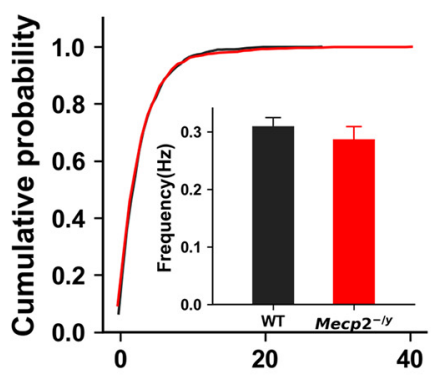

Inter-event interval(sec)

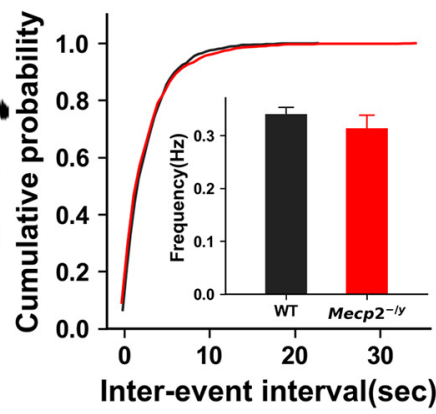

D
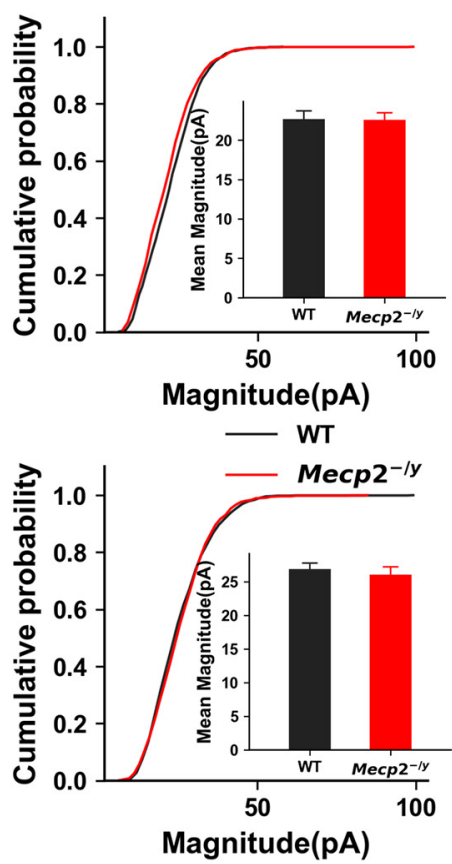
A

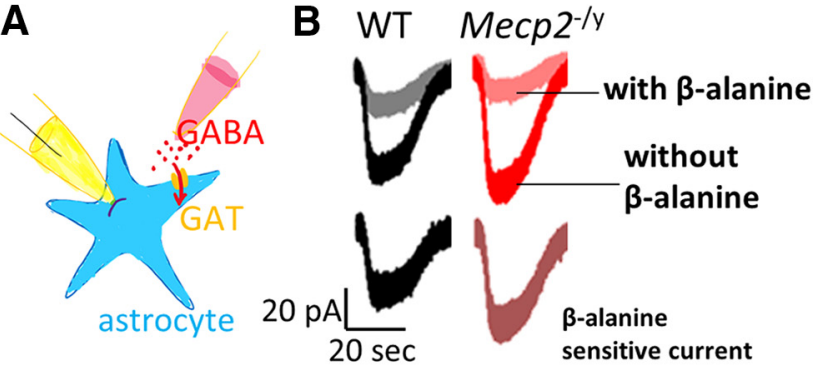

C

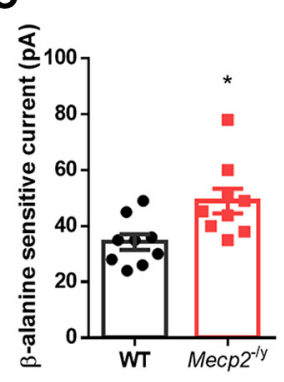

D

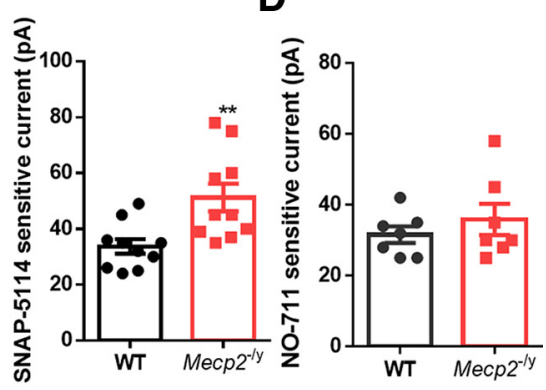

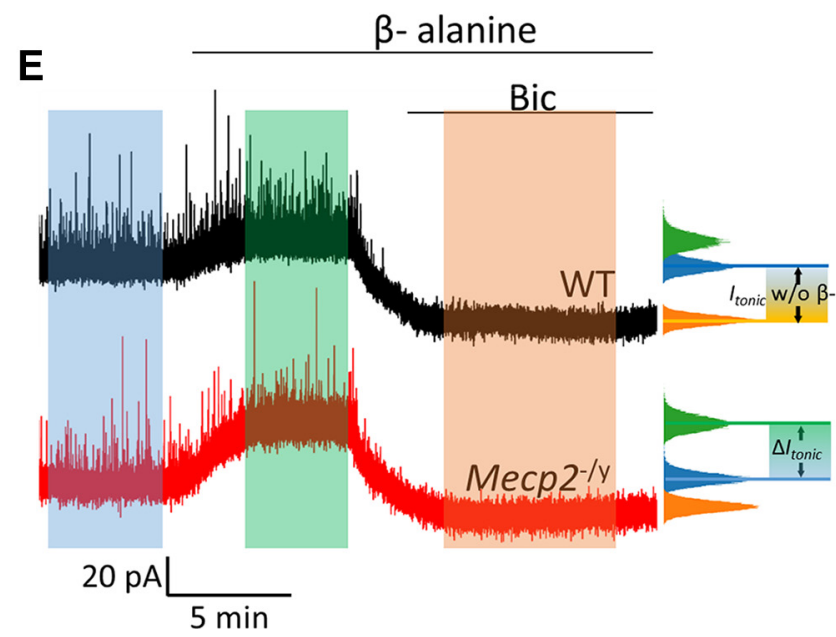

F

** $\beta$-alanine $\Delta l_{\text {tonic }} \quad$ * SNAP-5114 $\Delta l_{\text {tonic }}$

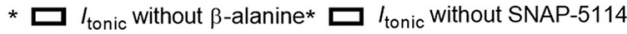

-

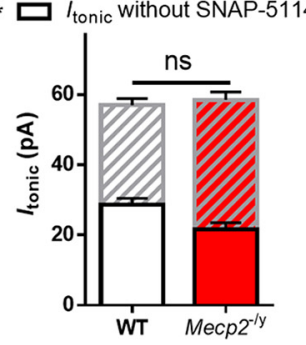

Figure 4. Enhanced astrocytic GAT3 activity underlying the reduced tonic GABA currents in Mecp2 KO hippocampal neurons. $\boldsymbol{A}$, Schematic drawing of the recording of the GAT3 current. $\boldsymbol{B}$, Representative traces of whole-cell patch-clamp recording of GABA induced currents with (gray and pink traces) or without (black and red traces) $\beta$-alanine in hippocampal astrocytes from WT and Mecp2 KO (Mecp2 $\left.{ }^{-/ y}\right)$ mice. The $\beta$-alanine-sensitive currents (the difference between these two currents) were shown at the bottom. Calibration: $20 \mathrm{pA}, 20 \mathrm{~s}$. $C$, Quantification of the amplitudes of $\beta$-alanine or SNAP5114-sensitive currents in hippocampal astrocytes from WT and Mecp2 K0 (Mecp2 ${ }^{-/ y}$ ) mice. D, Quantification of the amplitudes of N0-711, a GAT1 inhibitor, sensitive currents in hippocampal astrocytes from WT and Mecp2 KO (Mecp2 $2^{-1 y}$ ) mice. $\boldsymbol{E}$, Representative traces showing that the tonic inhibition was enhanced by $\beta$-alanine in both WT and Mecp2 KO (Mecp2 $\left.2^{-y}\right)$ CA1 neurons and how $I_{\text {tonic }}$ without inhibitor ( $\beta$-alanine or SNAP5114) and $\left.\Delta\right)_{\text {tonic }}$ in the presence of inhibitor ( $\beta$-alanine or SNAP5114) were measured. Calibration: $20 \mathrm{pA}, 5$ min. $\boldsymbol{F}$, Quantification of total $I_{\text {tonic }} I_{\text {tonic }}$ without inhibitor ( $\beta$-alanine or SNAP5114), and $\Delta I_{\text {tonic }}$ in the presence of inhibitor ( $\beta$-alanine or SNAP5114) in acute hippocampal slices prepared from WT and Mecp2 K0 (Mecp $\left.2^{-y}\right)$ mice. ${ }^{*} p<0.05{ }^{* *} p<0.01$ versus WT. ns, not significant.

$3 C, D)$. The GABA immunoreactivity was not significantly different between the WT and Mecp2 KO mice, either (WT: $100.0 \pm 5.76 \%, N=13$ randomly selected fields from 3 mice; Mecp2 KO: $94.86 .3 \pm 5.66 \%, N=13$ randomly selected fields from 3 mice; $t_{(24)}=0.64, p=0.53$; Fig. $\left.3 C, D\right)$, suggesting that the overall GABA content inside the cells remains unchanged in the Mecp2 KO hippocampus. To further look at GABA release probability, we measured evoked IPSCs (eIPSCs) in hippocampal CA1 pyramidal cells. The eIPSC paired-pulse ratios did not significantly differ between the two genotypes (WT: $1.561 \pm 0.072$, $N=12$ cells from 4 mice; Mecp2 KO: $1.658 \pm 0.060, N=12$ from 3 mice; $t_{(22)}=1.04, p=0.31$; Fig. $\left.3 E, F\right)$, indicating similar release probability of CA1 pyramidal neurons. However, the eIPSC decay rate was significantly faster in Mecp2 KO mice (WT: $21.80 \pm 1.90 \mathrm{~ms}, \quad N=10$ cells from 3 mice; Mecp2 KO: $16.93 \pm 0.93 \mathrm{~ms}, N=15$ cells from 3 mice; $t_{(23)}=2.55, p=0.02$; Fig. $3 F$ ), suggesting enhanced GABA uptake in the hippocampus of Mecp $2 \mathrm{KO}$ mice.

Given that the GABA release appears to be little changed and that the eIPSC decay rate is faster in those CA1 pyramidal neurons in the Mecp2 KO mice, the reduced ambient GABA level in the extracellular space might be because of increased GABA uptake mediated by GATs. The GATs rapidly clear GABA from the synaptic cleft, prevent GABA spillover (Overstreet and Westbrook, 2003), and control the ambient GABA concentration
(Scimemi, 2014). In the hippocampus, GAT3 is mainly expressed by astrocytes (Ribak et al., 1996). Interestingly, treatment of the Mecp2 KO slice with SNAP5114, a GAT3-selective antagonist, rescued the eIPSC decay rate change (Fig. $3 E, F$ ), which suggested a potential role of GAT3-mediated GABA uptake in phenotypes described so far.

We therefore examined the responsiveness of GAT3 by recording the currents mediated by its activity in response to GABA. Using GAT3-specific antagonists $\beta$-alanine and SNAP 5114 , we isolated GAT3 currents from astrocytes in acute hippocampal slices (Fig. 4A,B). These recordings showed that the GAT3 current from Mecp2 $\mathrm{KO}$ mice was significantly higher than that from the WT mice $(\beta$-alanine-sensitive current: WT: $34.22 \pm 2.803 \mathrm{pA}, N=9$ cells from 3 mice; Mecp2 KO: $49.00 \pm$ $4.40 \mathrm{pA}, N=9$ cells from 3 mice; $t_{(16)}=2.802, p=0.01$; SNAP 5114-sensitive current: WT: $33.70 \pm 2.612 \mathrm{pA}, N=10$ cells from 3 mice; Mecp2 KO: $51.20 \pm 4.968 \mathrm{pA}, N=10$ cells from 3 mice; $t_{(168)}=3.12, p=0.006$; Fig. $\left.4 C\right)$. Since astrocytes also express GABA transporter 1 (GAT1) (Chiu et al., 2002), we isolated GAT1-mediated current from astrocytes by using GAT1 selective inhibitor NO-711. The amplitude of NO-711-sensitive current was not significantly altered in Mecp2 $\mathrm{KO}$ mice (35.86 \pm $4.43 \mathrm{pA}, N=7$ cells from 3 mice) compared with WT mice $\left(31.57 \pm 2.32 \mathrm{pA}, N=7\right.$ cells from 3 mice; $\left.t_{(12)}=0.86, p=0.41\right)$, which suggested that the level of functional GAT1 was normal in Mecp2 KO mice. 

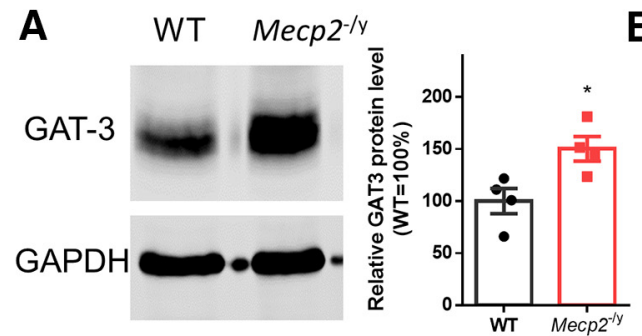

B
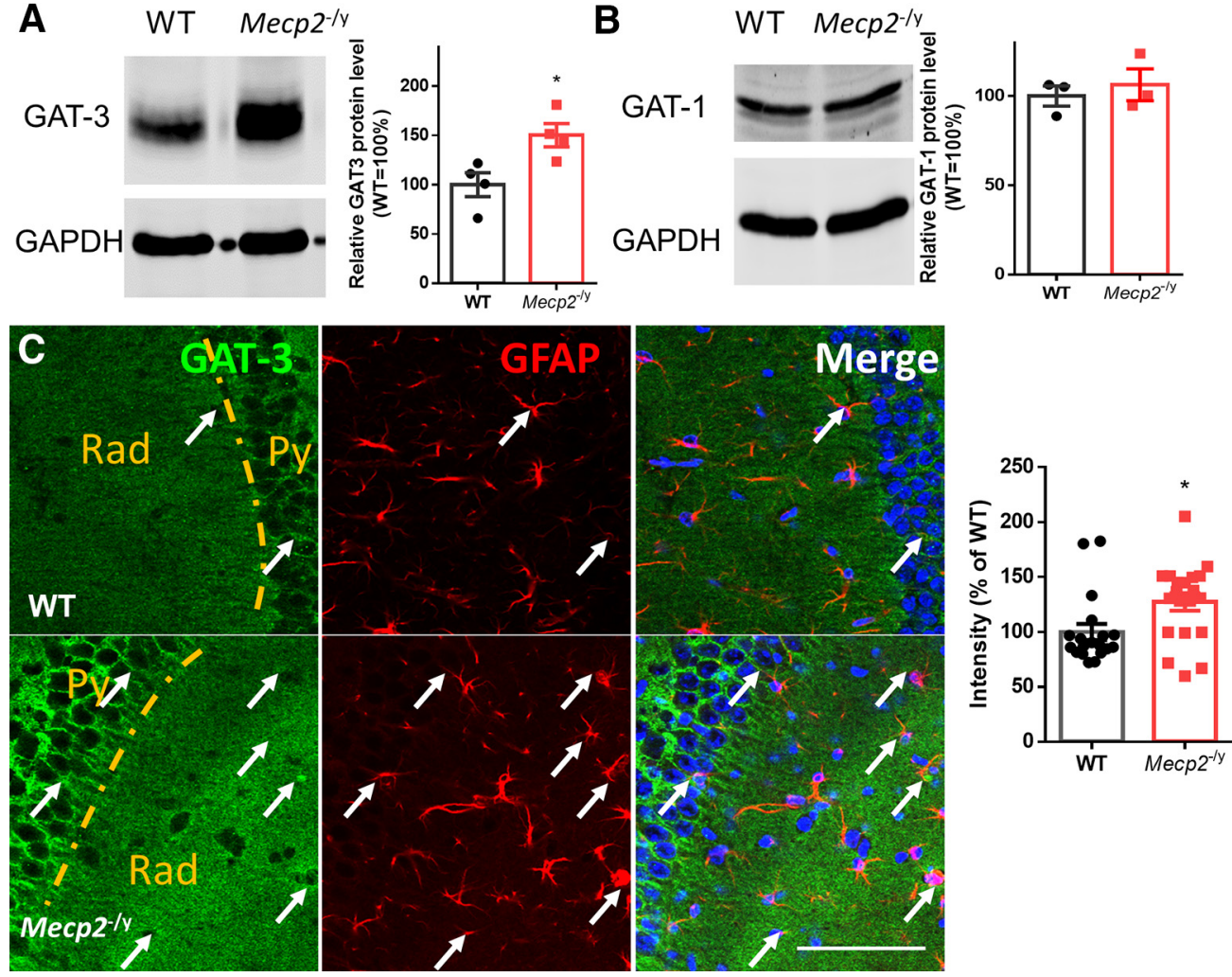

Figure 5. Upregulation of hippocampal GAT3 in Mecp2 K0 mice. $A$, Left, Representative images of Western blot of GAT3. Right, Quantification of the protein expression level of GAT3 in the hippocampus from WT and Mecp2 KO (Mecp2 ${ }^{-1 y}$ ) mice. B, Left, Representative images of Western blot of GAT1. Right, Quantification of the protein expression level of GAT1 in the hippocampus from WT and Mecp2 KO (Mecp2 ${ }^{-1 y}$ ) mice. C, Left, Representative GAT3 and GFAP costaining images from WT and Mecp2 K0 mice. Some GAT3 fluorescence signals are colocalized with GFAP ${ }^{+}$ astrocytic processes, which are indicated by arrows. Scale bar $=80 \mu \mathrm{m}$. Right, Quantification of GAT3 immunoreactivity in hippocampal sections from WT and Mecp2 K0 mice. ${ }^{*} p<0.05$ versus WT.

To determine the contribution of the enhanced GAT3 activity to the impairment of the tonic inhibition in the hippocampal CA1 pyramidal neurons from the Mecp2 KO mice, we superfused acute brain slices with $\beta$-alanine and SNAP 5114, respectively, while quantifying tonic inhibition. After a stable baseline was established, either $\beta$-alanine or SNAP 5114 was superfused, which was then followed up by bath application of bicuculline. Although both $\beta$-alanine and SNAP 5114 enhanced the tonic inhibition from the WT mice ( $\beta$-alanine $\Delta I_{\text {tonic }}: 25.00 \pm 2.44 \mathrm{pA}$, $N=7$ cells from 3 mice; SNAP $5114 \Delta I_{\text {tonic: }} 28.38 \pm 1.870 \mathrm{pA}$, $N=8$ cells from 3 mice), their respective effect on tonic inhibition was much greater in the Mecp $2 \mathrm{KO}$ mice ( $\beta$-alanine $\Delta I_{\text {tonic }}$ : $40.86 \pm 3.276 \mathrm{pA}, N=7$ cells from 3 mice; $t_{(12)}=3.88, p=0.002$; SNAP $5114 \Delta I_{\text {tonic }}: 36.90 \pm 2.193 \mathrm{pA}, N=10$ cells from 3 mice; $t_{(16)}=2.87, p=0.04$; Fig. $4 D, E$, stripes). As a result, the total $I_{\text {tonic }}$ was not different between WT and Mecp2 KO in the presence of either $\beta$-alanine or SNAP 5114. In another words, treating the Mecp2 KO slices with either $\beta$-alanine or SNAP 5114 rescued the phenotype of reduced tonic inhibition in hippocampal pyramidal neurons. These results suggest GAT3-mediated GABA uptake contributes to the observed tonic inhibition phenotype in the Mecp $2 \mathrm{KO}$ mice.

Next, we assessed the level of GAT3 protein in the Mecp2 KO hippocampus by Western blot. Our results showed that GAT3 was upregulated in the hippocampus from the Mecp2 KO mice $\left(164.3 \pm 25.98 \%, N=4\right.$ mice; $t_{(6)}=3.0, p=0.03$; Fig. $5 A)$. Consistent with the Western blot results, the immunoreactivity of GAT3 was also higher in the hippocampus of the Месp2 KO brain sections (WT: $100.0 \pm 7.31 \%, N=19$ brain sections from 3 mice; Mecp2 KO: $127.3 \pm 7.90 \%, N=20$ brain sections from 3 mice; Mann-Whitney $U$ test $=99, p=0.016$; Fig. $5 C$ ). In contrast, the protein expression level of GAT-1 did not change significantly in Mecp2 $\mathrm{KO}$ mice $(106.2 \pm 8.91 \%, N=3$ mice) compared with WT group $\left(100.0 \pm 5.65 \%, N=3\right.$ mice; $t_{(4)}=$ $0.59, p=0.59$; Fig. $5 B$ ).

Together, our electrophysiological and biochemical analyses suggest that astrocytes from the Mecp2 KO mice display excessive GAT3 activity, which could lead to reduced ambient GABA level in the extracellular space and contribute to the reduction of tonic inhibition in hippocampal CA1 pyramidal neurons.

\section{Reduced tonic inhibition in astrocytes-specific Mecp2 KO mice}

To determine whether deletion of Mecp2 specifically in astrocytes is sufficient to reproduce the phenotypes of impaired tonic inhibition in the hippocampus, we crossed male mice harboring a tamoxifen-inducible Cre recombinase transgene driven by the human astrocytic glial fibrillary acidic protein (hGFAP) promoter (hGFAP-creERT) with female mice possessing loxP sites flanking the Mecp2 gene $\left(M e c p 2^{\text {flox/flox }}\right)$. Half of the male offspring from this breeding that inherited both the hGFAPcreERT allele and the $M e c p 2^{\text {flox }}$ allele are referred to as Mecp $2^{f l o x / y}$; hGFAP-creERT mice. The remaining half of the male offspring from this breeding that inherited only the $M e c p 2^{\text {flox }}$ allele are referred to as Mecp $2^{\text {flox/y }}$ mice, which were used as control. Mice from both groups received daily tamoxifen injection for $7 \mathrm{~d}$. We first determined the specificity and efficiency of Cre recombinase activity in hGFAP-creERT mice that also carry the 

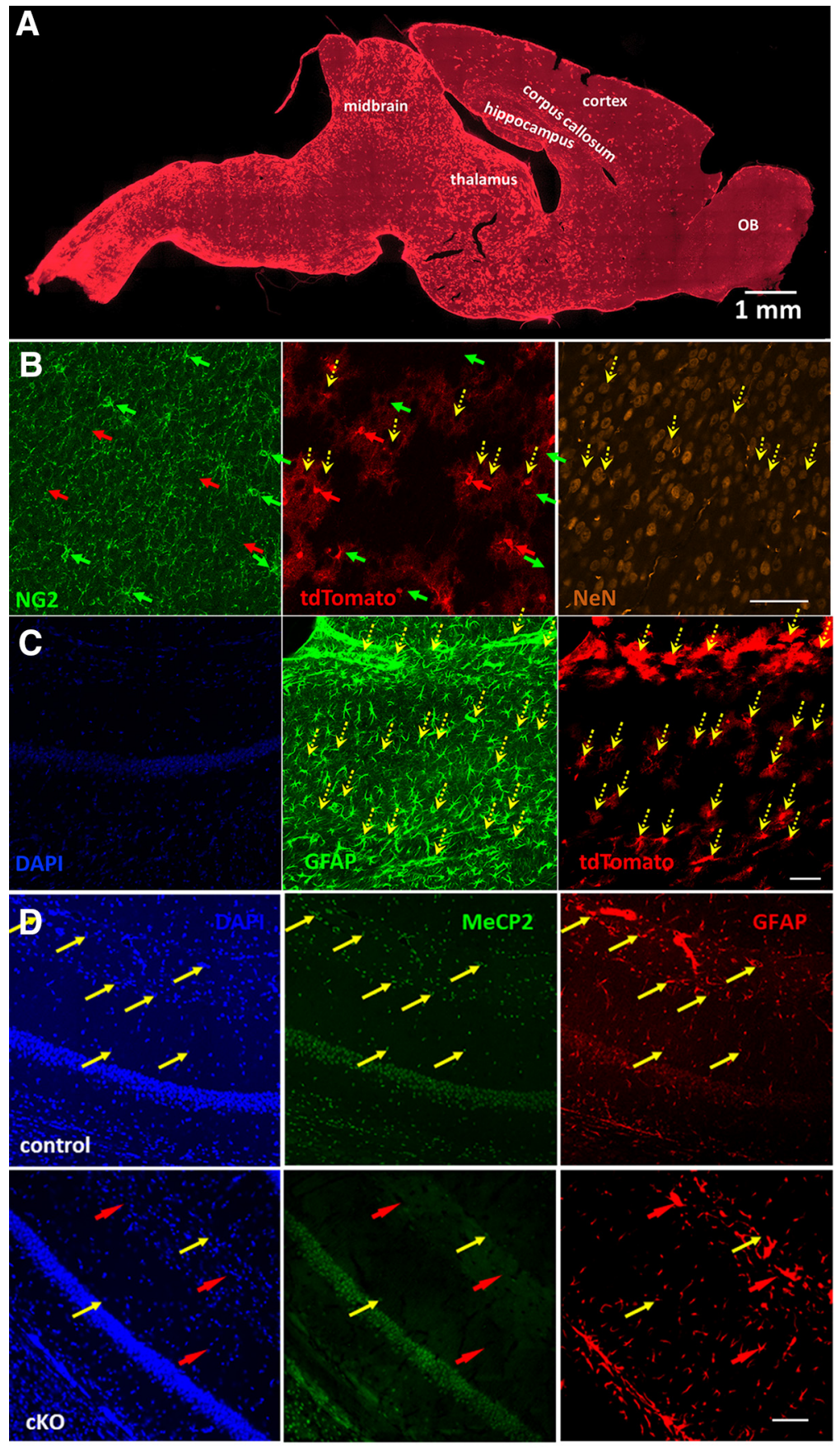

E
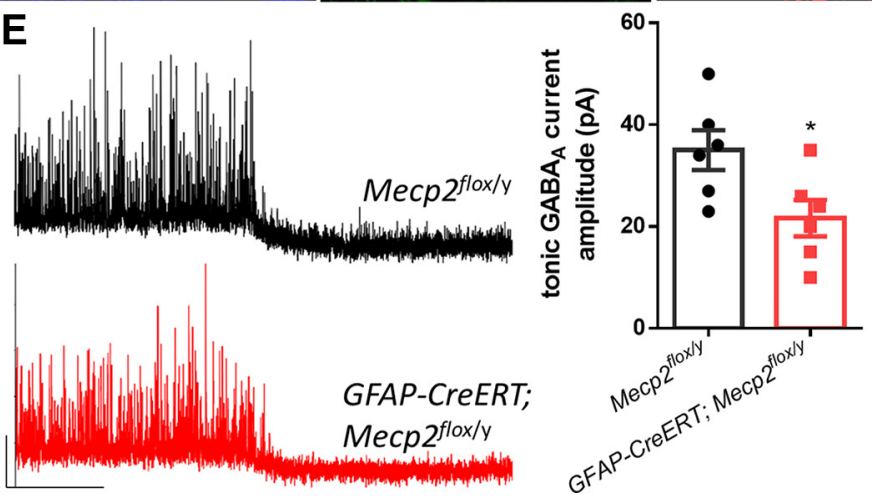

Figure 6. Reduced tonic GABA current in the hippocampus from astrocyte-specific Mecp2 K0 mice. $\boldsymbol{A}$, Representative image of tdTomato fluorescence on a sagittal section from an Ai14;hGFAP-creERT mouse injected with tamoxifen. $\boldsymbol{B}$, Representative confocal microscopy images showing NG2, NeuN, and tdTomato signals in the same section. Green, red, and yellow arrows indicate NG2-, tdTomato-, and NeuN-positive cells, respectively. Only few NG2-positive cells and no NeuN-positive cells showing tdTomato
Ai14 reporter allele 2 weeks after the completion of tamoxifen administration (Fig. 6A-C). More than $90 \%$ of tdTomato $^{+}$cells were $\mathrm{GFAP}^{+}$. In comparison, only $7 \%$ tdTomato $^{+}$cells were $\mathrm{NG}_{2}{ }^{+}$, and no tdTomato ${ }^{+}$cell was $\mathrm{NeuN}^{+}$. These data suggest that, in the short time window (2 weeks) after tamoxifen induction, the vast majority of the Cre-expressing cells are astrocytes, and no neurons express Cre, in the hippocampus. When we next assessed the specificity and efficiency of Mecp2 deletion in the hippocampus of the Mecp $2^{f l o x / y}$; hGFAP-creERT mice 2 weeks after the completion of tamoxifen administration, we observed a high percentage of $\mathrm{MeCP} 2$-negative and GFAP-positive astrocytes in hippocampus $(85 \pm 5.8 \%$ ) (Fig. $6 D$ ).

We next recorded the tonic inhibition currents from these mice. Compared with tamoxifen-treated control mice, tamoxifen-treated Mecp $2^{f l o x / y}$; hGFAP-creERT mice showed decreased tonic current in hippocampal CA1 pyramidal neurons (Fig. $6 E$; control: $35.00 \pm 3.873 \mathrm{pA}, N=6$ cells from 2 mice; mutant: $21.67 \pm 3.575 \mathrm{pA}, N=$ 6 cells from 2 mice; $t_{(10)}=2.5, p=$ 0.03). Moreover, the difference is comparable with the difference between WT and Mecp2 KO mice (compare Fig. $6 E$ with Fig. $1 B$ ), indicating that astrocyte-specific Mecp2 deletion is sufficient to recapitulate the phenotype of impaired tonic inhibition observed in Mecp2 KO mice, and further highlighting the important role of astrocytes in the development of this new neuronal phenotype in RTT.

\footnotetext{
fluorescence. C, Representative confocal microscopy images showing colocalization of GFAP and tdTomato signals in a hippocampal section. Yellow arrows indicate tdTomato and GFAP double-positive cells. $\boldsymbol{D}$, Representative confocal microscopy images of hippocampal sections costained with anti-MeCP2 and antiGFAP antibodies. Top, Images from a GFAP-CreERT; Mecp $2^{\text {floxy }}$ mouse injected with tamoxifen (cKO). Bottom, Images from a Mecp $2^{\text {flox/y }}$ mouse injected with tamoxifen (control). Yellow arrows indicate some $\mathrm{GFAP}^{+}$cells showing MeCP2 staining. Red arrows indicate some $\mathrm{GFAP}^{+}$cells without MeCP2 fluorescence. There are many MeCP2-positive astrocytes in the control mouse but not in the cKO mouse. Scale bar, $50 \mu \mathrm{m}$. $\boldsymbol{E}$, Left, Representative traces of tonic GABA current recordings from control (Mecp $2^{f l o x / y}$ ) and astrocyte-specific Mecp2 KO (cKO, GFAP-CreERT: $M e c p 2^{\text {flox/y }}$ ) mice. Right, Quantification of the amplitude of the tonic inhibition from control and $\mathrm{CKO}$ mice. ${ }^{*} p<0.05$ versus control.
} 
Inhibiting GAT3 rescued the hyperexcitability and increased seizure susceptibility in Mecp2 $\mathrm{KO}$ mice and extended the lifespan in Mecp2 $\mathrm{KO}$ mice

Tonic $\mathrm{GABA}_{\mathrm{A}} \mathrm{R}$-mediated conductance regulates the neuronal excitability by decreasing total membrane input resistance (Hamann et al., 2002; Stell et al., 2003). To determine whether the excitability of hippocampal CA1 pyramidal neurons is altered by the decreased tonic inhibition observed in Mecp $2 \mathrm{KO}$ mice, we evaluated resting membrane potentials and firing rate in response to current injection using whole-cell current-clamp recordings. The resting membrane potential of neurons from WT and Mecp2 KO mice was similar (WT: $-68 \pm 2.6 \mathrm{mV}, N=7$ cells from 3 mice; Mecp2 KO: $-66 \pm 3.2 \mathrm{mV}, N=7$ cells from 3 mice; $\left.t_{(12)}=0.49, p=0.65\right)$. However, hippocampal CA1 pyramidal neurons of Mecp2 $\mathrm{KO}$ mice required lower current injection to fire action potentials. The firing rate was higher in Mecp2 KO mice in response to the same current injection, indicating the hyperexcitability in Mecp2 KO mice (Fig. 7A; two-way ANOVA, $\left.F_{(1,10)}=14.03, p=0.004\right)$. To demonstrate whether the hyperexcitability is mediated by the impairment of tonic inhibition, the GAT3 antagonist $\beta$-alanine was added to the bath solution. In the presence of $\beta$-alanine, the firing rate of hippocampal neurons from Mecp2 $\mathrm{KO}$ mice was significantly decreased (Fig. 7A; two-way ANOVA, $\left.F_{(1,11)}=11.62, p=0.006\right)$.

RTT patients and Mecp2 KO mice are characterized by their susceptibility to seizure; $80 \%-90 \%$ of RTT patients have seizures (Jian et al., 2007), and spontaneous seizures have also been reported in RTT mice (D'Cruz et al., 2010; McLeod et al., 2013). To assess the contribution of the impairment of tonic inhibition to seizure susceptibility in Mecp2 $\mathrm{KO}$ mice, we used an in vitro seizure model by superfusing hippocampal slices with $0 \mathrm{Mg}^{2+}$ aCSF to induce epileptic activities in both genotypes: WT and Mecp2 KO (Fig. 7B). Compared with the WT slices, epileptiform activity in Mecp2 $\mathrm{KO}$ slices had shorter latency (Fig. 7C; WT: $6.66 \pm 0.43 \mathrm{~min}, N=10$ cells from 4 mice; Mecp2 KO: $4.82 \pm$ $0.50 \mathrm{~min}, N=9$ cells from 4 mice; $t_{(17)}=2.78, p=0.01$ ), higher frequency (Fig. 7C; WT: $3.83 \pm 0.66 \mathrm{~min}^{-1}, N=10$ cells from 4 mice; Mecp2 KO: $6.00 \pm 0.44 \mathrm{~min}^{-1}, N=9$ cells from 4 mice; $t_{(17)}=2.61, p=0.02$ ), longer duration (Fig. 7C; WT: $615 \pm$ $29.86 \mathrm{~ms}, N=10$ cells from 4 mice; Mecp2 KO: $783.33 \pm$ $29.50 \mathrm{~ms}, N=9$ cells from 4 mice; $t_{(17)}=4.0, p=0.009$ ), and higher amplitude (Fig. 7C; WT: $291 \pm 21 \mathrm{pA}, N=10$ cells from 4 mice; Mecp2 KO: $351 \pm 14 \mathrm{pA}, N=9$ cells from 4 mice; $t_{(17)}=$ $2.3, p=0.03)$. More importantly, $\beta$-alanine application significantly reduced epileptiform activity in Mecp2 KO mice (Fig. 7C) by increasing the latency $(6.44 \pm 0.51 \mathrm{~min}, N=8$ cells from 4 mice; $\left.t_{(15)}=2.23, p=0.04\right)$, decreasing the frequency $(4.33 \pm$ $0.42 \mathrm{~min}^{-1}, N=8$ cells from 4 mice; $\left.t_{(15)}=2.67, p=0.02\right)$, decreasing the duration $(550 \pm 6.0 \mathrm{~ms}, N=8$ cells from 4 mice; $\left.t_{(15)}=4.74, p=0.0003\right)$, and decreasing the amplitude (303 \pm $14 \mathrm{pA}, N=8$ cells from 4 mice; $\left.t_{(15)}=2.4, p=0.03\right)$. These results indicate that the enhanced GAT activity and the resulting impairment of tonic inhibition could contribute to increased seizure susceptibility in Mecp2 $\mathrm{KO}$ mice.

To determine the disease relevance of increased GAT3 activity, we tested whether inhibiting the activity of GAT3 could slow down disease progression in the Mecp2 KO mice. Male Mecp2 $\mathrm{KO}$ mice were given daily intraperitoneal injection of either vehicle or $\beta$-alanine, and scored weekly for signs of the disease phenotype using a well-established observational severity score (Guy et al., 2007). Both vehicle- and $\beta$-alanine-treated mice developed increasing severity scores over time from postnatal week 5 to the time of death. However, mice injected with $\beta$-alanine had less severe observational scores than mice receiving vehicle treatment (Fig. 7D; 15 mice in each treatment group, two-way ANOVA with post hoc Holm-Sidak method, $p=0.024$ ). Survival analysis indicated that, compared with vehicle-treated Mecp2 KO mice, $\beta$-alanine-treated Mecp2 KO mice lived significantly longer (15 mice in each treatment group, log-rank (Mantel-Cox) test, $p=0.021$; Fig. $7 E$ ).

\section{Discussion}

Altered tonic inhibition has been observed in animal models of a wide range of neurologic diseases, including Fragile X syndrome (Curia et al., 2009; Zhang et al., 2017), Angelman syndrome (Egawa et al., 2012), autism (Bridi et al., 2017), and others (Stell et al., 2003; Cope et al., 2009; Gupta et al., 2012; Wójtowicz et al., 2013; Wu et al., 2014; Miguelez et al., 2018). Among the disease models displaying abnormal tonic inhibition, different mechanisms are involved, including changes in activity or expression of GABA receptors, GATs, or glutamic acid decarboxylase, all of which lead to the impairment or argumentation of tonic GABA inhibition. In our current study, we discovered a novel phenotype of reduced tonic inhibition in hippocampal CA1 pyramidal neurons in the RTT mice. Moreover, we observed a concurrent phenotype of increased GAT (GAT3) expression and activity in the neighboring astrocytes. Thus, not only does our study add RTT to the long list of neurologic diseases affected by altered tonic inhibition, it also demonstrates another cell-autonomous phenotype in RTT astrocyte and its consequence on RTT neurons. Those data further advance our understanding of the multicellular nature of RTT pathogenesis.

In a separate study, we recently reported increased spontaneous $\mathrm{Ca}^{2+}$ activity and elevated cytosolic $\mathrm{Ca}^{2+}$ concentration in Mecp2 mutant astrocytes (Dong et al., 2018). Since $\mathrm{Ca}^{2+}$ is known to regulate the functional expression of GAT3 under some conditions (Shigetomi et al., 2011), it would be a natural next step to determine whether those two phenotypes are connected in RTT astrocytes. To that end, we recorded the tonic inhibitory currents from the Mecp $2 \mathrm{KO}$ mice while manipulating cytosolic $\mathrm{Ca}^{2+}$ by intracellular BAPTA (10 mM) dialysis of single astrocytes. Because of the gap junctional coupling existed among astrocytes, such treatment would block spontaneous $\mathrm{Ca}^{2+}$ activity and reduce the cytosolic $\mathrm{Ca}^{2+}$ in all connected astrocytes. Under this condition, reduced tonic inhibition currents were still detected in Mecp2 KO neurons, when compared with that in the WT neurons (data not shown), indicating no direct connection between our previous finding of abnormal $\mathrm{Ca}^{2+}$ homeostasis in RTT astrocytes and our current finding of increased GAT3 activity in RTT astrocytes. In addition, we observed no reduction of tonic inhibition in WT neurons by elevating cytosolic $\mathrm{Ca}^{2+}$ in astrocytes through " $\mathrm{Ca}^{2+}$ clamping" (data not shown). At more detailed level, it should be noted that past work linking $\mathrm{Ca}^{2+}$ with GAT3 (Shigetomi et al., 2011) showed that reduced, but not increased, $\mathrm{Ca}^{2+}$ activity is important to the regulation of GAT3 expression and tonic inhibition. Together, our unpublished results and past work in other laboratories are consistent with the notion that the phenotypes of increased GAT3 level/activity and reduced tonic inhibition cannot be explained by the phenotype of heightened $\mathrm{Ca}^{2+}$ activity and elevated $\mathrm{Ca}^{2+}$ concentration in RTT astrocytes.

It is interesting to note that our reported findings are specific for the hippocampus, as Western blot analysis and whole-cell patch-clamp recordings detected no change in GAT3 expression or tonic inhibition in the cortex (data not shown). Adding to the 
A
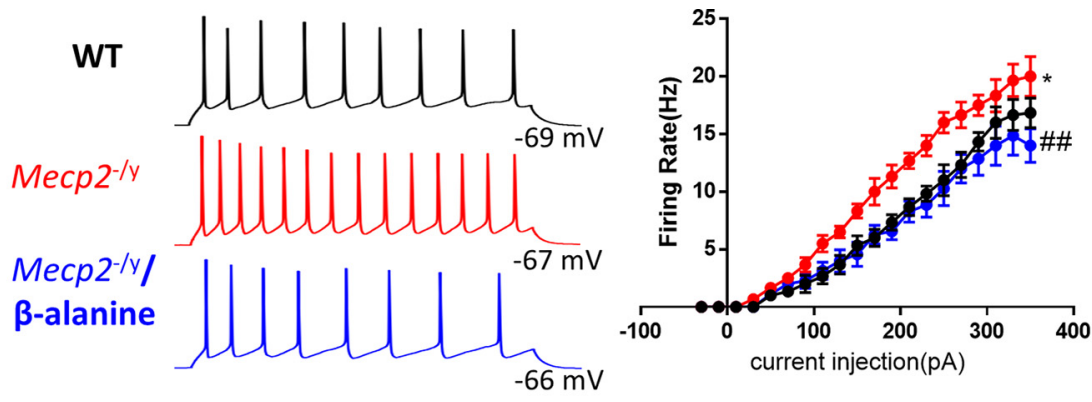

B
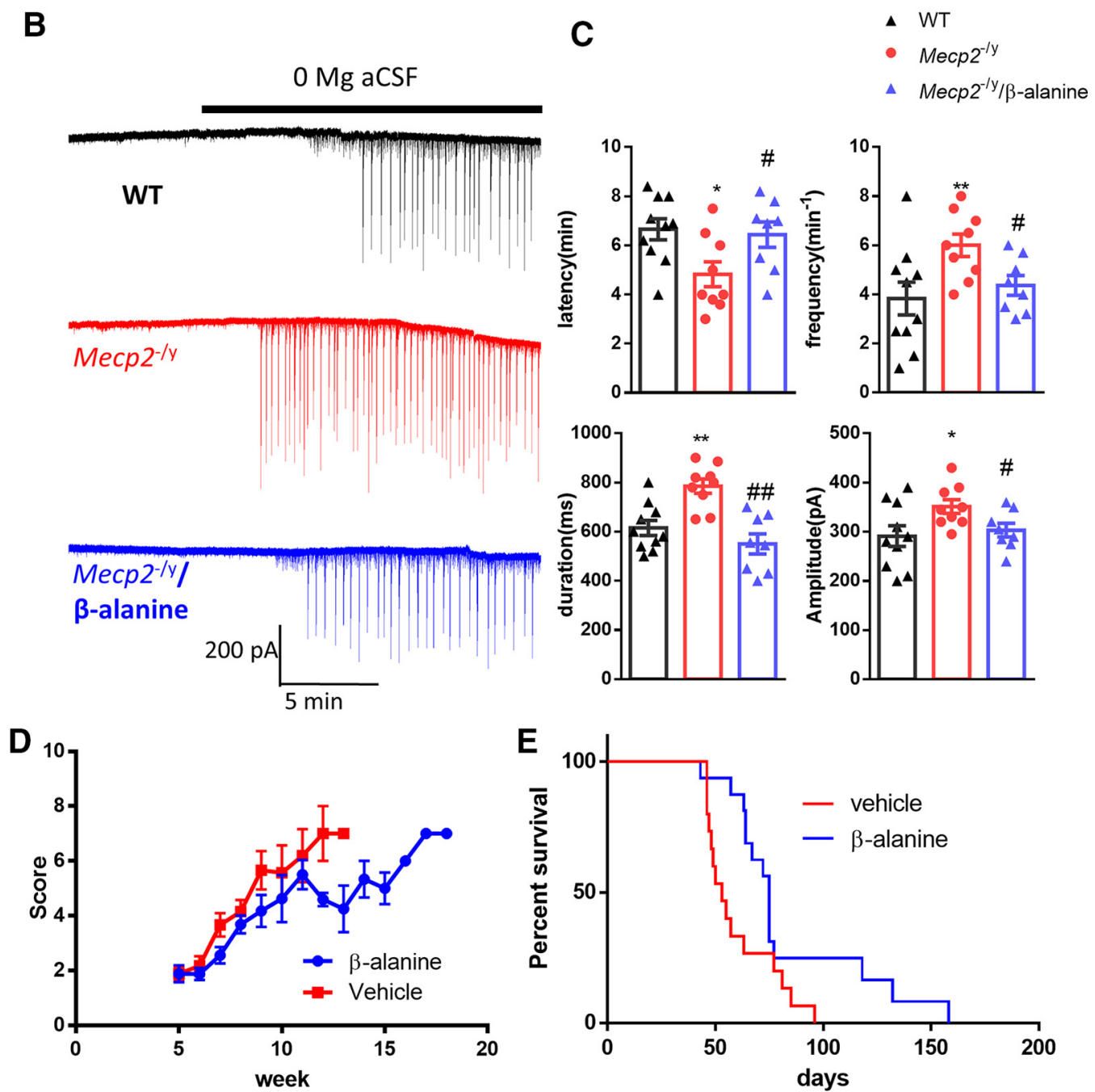

Figure 7. Inhibition of GAT3 rescued hyperexcitability phenotypes in acute hippocampal slices from the Mecp2 K0 mice and alleviated symptoms in Mecp2 K0 mice. $A$, Left, Representative traces showing current injection-evoked action potentials recorded from hippocampal pyramidal neurons in WT (black), Mecp2 K0 (Mecp2- ${ }^{-1 y}$, red), and Mecp2 K0 in the presence of $\beta$-alanine (blue). Right, Quantification of the firing rate of hippocampal neurons in response to different current steps. ${ }^{*} p<0.05$ versus WT. ${ }^{\# \#} p<0.01$ versus Mecp2 ${ }^{-1 y}$. B, Representative traces showing epileptiform activity induced by $0 \mathrm{Mg}$ aCSF in hippocampal slices from WT (top) and Mecp2 KO (Mecp $2^{-1 y}$ ) mice in the absence (middle) or presence of $\beta$-alanine (bottom). $C$, Quantification of the latency, frequency, duration, and amplitude of the epileptiform activity. The latency is defined as the time elapsed between the application of $0 \mathrm{Mg}$ aCSF and the onset of epileptiform activity. ${ }^{*} p<0.05,{ }^{* *} p<0.01$ versus WT. ${ }^{\#} p<0.05$ versus Mecp2 KO. D. The phenotypic severity scores of male Mecp2 K0 (Mecp2 ${ }^{-1 y}$ ) mice receiving daily injection of vehicle or $\beta$-alanine. $\boldsymbol{E}$, Survival curves of male Mecp2 $\mathrm{KO}\left(\right.$ Mecp2 $\left.{ }^{-/ y}\right)$ mice receiving daily injection of vehicle or $\beta$-alanine.

notion of brain region-specific phenotypes in tonic inhibition, previous research showed augmented tonic inhibitory currents in locus ceruleus neurons in RTT mice (Zhong et al., 2015). In that study, the authors described an upregulation of the GABA $\delta$ subunit in LC neurons. In another study, Calfa et al. (2015) demonstrated that the immunoreactivity of GABA $\alpha 1$ subunit decreased in the hippocampal CA3 pyramidal cell body layer of $M e c p 2^{-l y}$ mice. They also found a decrease in amplitude of
mIPSCs in CA3 pyramidal neurons. In contrast, we found no change in the functional level of $\mathrm{GABA}_{\mathrm{A}}$ receptor in hippocampal CA1 pyramidal neurons in RTT mice because GABA-evoked currents from WT and Mecp2 KO mice have similar magnitudes. These studies highlight the issue of regional specificity in phenotypes observed in Mecp2 $\mathrm{KO}$ mice, which appears to arise from different molecular underpinnings. This is not surprising given that $\mathrm{MeCP} 2$ is expressed in almost all the major cell types in the 
brain, and that the loss of MeCP2 function can lead to different gene expression and functional changes in different cell types across different regions of the brain.

Although GAT3 is reported to be expressed mainly in astrocytes, it is also possible that GAT3 is aberrantly expressed in MeCP2-deficient hippocampal neurons. We recorded SNAP5114sensitive current from neurons, whose amplitude was very small. Nonetheless, there was no significant difference between WT and Mecp $2 \mathrm{KO}$ mice in the amplitude of these currents (data not shown), which indicates little contribution of neuronal GAT3 to our observed phenotype in ambient GABA level.

In addition to GAT3, GAT1 is another subtype of GAT important for the regulation of ambient GABA level. GAT1 is expressed at a high density and in vicinity to synaptic GABA release sites, mostly in presynaptic boutons of interneurons and astroglial processes (Minelli et al., 1996; Chiu et al., 2002). Since Western blot analysis of GAT1 expression in hippocampus tissue showed similar GAT1 protein levels between WT and Mecp $2 \mathrm{KO}$ mice, we focused our analysis on GAT3. Using GAT3-selective antagonist $\beta$-alanine, our study assessed the contribution of the impaired tonic inhibition to RTT at different levels, including intrinsic neuronal excitability at the cellular level, epileptiform activity at the network level, and disease progression in the whole animal. Our results show $\beta$-alanine normalized the hyperexcitability phenotype (both intrinsic excitability and susceptibility to seizure) in Mecp2 KO mice and delayed the onset of symptoms of RTT. Because the Mecp2 gene is located on the $\mathrm{X}$ chromosome, all female Mecp2 heterozygous $\left(M e c p 2^{+/-}\right)$mice are at different levels of mosaicism of somatic cells with or without MeCP2. In comparison, the male Mecp2 KO mice present a cleaner biological system to perform the initial characterization of a new phenotype and its associated mechanisms. For that reason, all mice used in this study are male. However, we fully recognize that the female Mecp2 heterozygous mice more closely resemble the female RTT patients, and will further this line of research in the female mice. As a first step, we already observed the same tonic inhibition phenotype in the hippocampus of female Mecp2 heterozygous mice (data not shown).

In conclusion, we report a novel phenotype of reduced tonic inhibition in hippocampal CA1 pyramidal neurons in the RTT mice, reveal a potential mechanism of increased GAT expression/activity in the neighboring astrocytes, describe a disease-relevant consequence in hyperexcitability, and provide preliminary evidence that targeting this phenotype may slow down disease progression in RTT mice. These results help our understanding of the disease etiology and identify a new therapeutic target for treating the disease.

\section{References}

Amir RE, Van den Veyver IB, Wan M, Tran CQ, Francke U, Zoghbi HY (1999) Rett syndrome is caused by mutations in X-linked MECP2, encoding methyl-CpG-binding protein 2. Nat Genet 23:185-188.

Bai D, Zhu G, Pennefather P, Jackson MF, MacDonald JF, Orser BA (2001) Distinct functional and pharmacological properties of tonic and quantal inhibitory postsynaptic currents mediated by gamma-aminobutyric acid (A) receptors in hippocampal neurons. Mol Pharmacol 59:814-824.

Brickley SG, Mody I (2012) Extrasynaptic GABA(A) receptors: their function in the CNS and implications for disease. Neuron 73:23-34.

Bridi MS, Park SM, Huang S (2017) Developmental disruption of GABARmeditated inhibition in Cntnap2 KO Mice. eNeuro 4:ENEURO.016217.2017.

Bright DP, Smart TG (2013) Methods for recording and measuring tonic GABAA receptor-mediated inhibition. Front Neural Circuits 7:193.
Calfa G, Li W, Rutherford JM, Pozzo-Miller L (2015) Excitation/inhibition imbalance and impaired synaptic inhibition in hippocampal area CA3 of Mecp2 knockout mice. Hippocampus 25:159-168.

Caraiscos VB, Elliott EM, You-Ten KE, Cheng VY, Belelli D, Newell JG, Jackson MF, Lambert JJ, Rosahl TW, Wafford KA, MacDonald JF, Orser BA (2004) Tonic inhibition in mouse hippocampal CA1 pyramidal neurons is mediated by alpha5 subunit-containing gamma-aminobutyric acid type A receptors. Proc Natl Acad Sci USA 101:3662-3667.

Chao HT, Chen H, Samaco RC, Xue M, Chahrour M, Yoo J, Neul JL, Gong S, Lu HC, Heintz N, Ekker M, Rubenstein JL, Noebels JL, Rosenmund C, Zoghbi HY (2010) Dysfunction in GABA signalling mediates autism-like stereotypies and Rett syndrome phenotypes. Nature 468:263-269.

Chen RZ, Akbarian S, Tudor M, Jaenisch R (2001) Deficiency of methylCpG binding protein-2 in CNS neurons results in a Rett-like phenotype in mice. Nat Genet 27:327-331.

Chiu CS, Jensen K, Sokolova I, Wang D, Li M, Deshpande P, Davidson N, Mody I, Quick MW, Quake SR, Lester HA (2002) Number, density, and surface/cytoplasmic distribution of GABA transporters at presynaptic structures of knock-in mice carrying GABA transporter subtype 1-green fluorescent protein fusions. J Neurosci 22:10251-10266.

Cope DW, Di Giovanni G, Fyson SJ, Orbán G, Errington AC, Lorincz ML, Gould TM, Carter DA, Crunelli V (2009) Enhanced tonic GABAA inhibition in typical absence epilepsy. Nat Med 15:1392-1398.

Cope DW, Hughes SW, Crunelli V (2005) GABAA receptor-mediated tonic inhibition in thalamic neurons. J Neurosci 25:11553-11563.

Curia G, Papouin T, Séguéla P, Avoli M (2009) Downregulation of tonic GABAergic inhibition in a mouse model of fragile X syndrome. Cereb Cortex 19:1515-1520.

D'Cruz JA, Wu C, Zahid T, El-Hayek Y, Zhang L, Eubanks JH (2010) Alterations of cortical and hippocampal EEG activity in MeCP2-deficient mice. Neurobiol Dis 38:8-16.

Dong Q, Liu Q, Li R, Wang A, Bu Q, Wang KH, Chang Q (2018) Mechanism and consequence of abnormal calcium homeostasis in Rett syndrome astrocytes. eLife 7:e33417.

Durand S, Patrizi A, Quast KB, Hachigian L, Pavlyuk R, Saxena A, Carninci P, Hensch TK, Fagiolini M (2012) NMDA receptor regulation prevents regression of visual cortical function in the absence of Mecp2. Neuron 76:1078-1090

Egawa K, Kitagawa K, Inoue K, Takayama M, Takayama C, Saitoh S, Kishino T, Kitagawa M, Fukuda A (2012) Decreased tonic inhibition in cerebellar granule cells causes motor dysfunction in a mouse model of Angelman syndrome. Sci Transl Med 4:163ra157.

El-Khoury R, Panayotis N, Matagne V, Ghata A, Villard L, Roux JC (2014) GABA and glutamate pathways are spatially and developmentally affected in the brain of Mecp2-deficient mice. PLoS One 9:e92169.

Farrant M, Nusser Z (2005) Variations on an inhibitory theme: phasic and tonic activation of GABA(A) receptors. Nat Rev Neurosci 6:215-229.

Gupta A, Elgammal FS, Proddutur A, Shah S, Santhakumar V (2012) Decrease in tonic inhibition contributes to increase in dentate semilunar granule cell excitability after brain injury. J Neurosci 32:2523-2537.

Guy J, Hendrich B, Holmes M, Martin JE, Bird A (2001) A mouse Mecp2null mutation causes neurological symptoms that mimic Rett syndrome. Nat Genet 27:322-326.

Guy J, Gan J, Selfridge J, Cobb S, Bird A (2007) Reversal of neurological defects in a mouse model of Rett syndrome. Science 315:1143-1147.

Hamann M, Rossi DJ, Attwell D (2002) Tonic and spillover inhibition of granule cells control information flow through cerebellar cortex. Neuron 33:625-633.

He L, Liu N, Cheng T, Chen X, Li Y, Shu Y, Qiu Z, Zhang X (2014) Conditional deletion of Mecp2 in parvalbumin-expressing GABAergic cells results in the absence of critical period plasticity. Nat Commun 5:5036.

Ito-Ishida A, Ure K, Chen H, Swann JW, Zoghbi HY (2015) Loss of MeCP2 in parvalbumin- and somatostatin-expressing neurons in mice leads to distinct Rett syndrome-like phenotypes. Neuron 88:651-658.

Jian L, Nagarajan L, de Klerk N, Ravine D, Christodoulou J, Leonard H (2007) Seizures in Rett syndrome: an overview from a one-year calendar study. Eur J Paediatr Neurol 11:310-317.

Krishnan K, Lau BY, Ewall G, Huang ZJ, Shea SD (2017) MECP2 regulates cortical plasticity underlying a learned behaviour in adult female mice. Nat Commun 8:14077. 
Krishnan K, Wang BS, Lu J, Wang L, Maffei A, Cang J, Huang ZJ (2015) $\mathrm{MeCP} 2$ regulates the timing of critical period plasticity that shapes functional connectivity in primary visual cortex. Proc Natl Acad Sci USA 112: E4782-E4791.

Lee E, Lee J, Kim E (2017) Excitation/inhibition imbalance in animal models of autism spectrum disorders. Biol Psychiatry 81:838-847.

McLeod F, Ganley R, Williams L, Selfridge J, Bird A, Cobb SR (2013) Reduced seizure threshold and altered network oscillatory properties in a mouse model of Rett syndrome. Neuroscience 231:195-205.

Miguelez C, Boué-Grabot E, Panatier A, Sebastiao A, Vaz S, CristóvãoFerreira S, Paredes-Rodriguez E, Martinez A, Morin S, Baufreton J, Chazalon M (2018) GAT-3 dysfunction generates tonic inhibition in external globus pallidus neurons in parkinsonian rodents. Cell Rep 23:1678-1690.

Minelli A, DeBiasi S, Brecha NC, Zuccarello LV, Conti F (1996) GAT-3, a high-affinity GABA plasma membrane transporter, is localized to astrocytic processes, and it is not confined to the vicinity of GABAergic synapses in the cerebral cortex. J Neurosci 16:6255-6264.

Nelson SB, Valakh V (2015) Excitatory/inhibitory balance and circuit homeostasis in autism spectrum disorders. Neuron 87:684-698.

Noutel J, Hong YK, Leu B, Kang E, Chen C (2011) Experience-dependent retinogeniculate synapse remodeling is abnormal in MeCP2-deficient mice. Neuron 70:35-42.

Nusser Z, Mody I (2002) Selective modulation of tonic and phasic inhibitions in dentate gyrus granule cells. J Neurophysiol 87:2624-2628.

Overstreet LS, Westbrook GL (2003) Synapse density regulates independence at unitary inhibitory synapses. J Neurosci 23:2618-2626.

Porcello DM, Huntsman MM, Mihalek RM, Homanics GE, Huguenard JR (2003) Intact synaptic GABAergic inhibition and altered neurosteroid modulation of thalamic relay neurons in mice lacking delta subunit. J Neurophysiol 89:1378-1386.

Ribak CE, Tong WM, Brecha NC (1996) GABA plasma membrane transporters, GAT-1 and GAT-3, display different distributions in the rat hippocampus. J Comp Neurol 367:595-606.

Santhakumar V, Hanchar HJ, Wallner M, Olsen RW, Otis TS (2006) Contributions of the GABAA receptor alpha6 subunit to phasic and tonic inhibition revealed by a naturally occurring polymorphism in the alpha6 gene. J Neurosci 26:3357-3364.

Scimemi A (2014) Structure, function, and plasticity of GABA transporters. Front Cell Neurosci 8:161.

Semyanov A, Walker MC, Kullmann DM (2003) GABA uptake regulates cortical excitability via cell type-specific tonic inhibition. Nat Neurosci 6:484-490.

Shigetomi E, Tong X, Kwan KY, Corey DP, Khakh BS (2011) TRPA1 channels regulate astrocyte resting calcium and inhibitory synapse efficacy through GAT-3. Nat Neurosci 15:70-80.

Stell BM, Brickley SG, Tang CY, Farrant M, Mody I (2003) Neuroactive steroids reduce neuronal excitability by selectively enhancing tonic inhibition mediated by delta subunit-containing GABAA receptors. Proc Natl Acad Sci USA 100:14439-14444.

Ure K, Lu H, Wang W, Ito-Ishida A, Wu Z, He LJ, Sztainberg Y, Chen W, Tang J, Zoghbi HY (2016) Restoration of Mecp2 expression in GABAergic neurons is sufficient to rescue multiple disease features in a mouse model of Rett syndrome. eLife 5:e14198.

Wójtowicz AM, Grantyn R, Dvorzhak A, Semtner M (2013) Reduced tonic inhibition in striatal output neurons from Huntington mice due to loss of astrocytic GABA release through GAT-3. Front Neural Circuits 7:188.

Wu Z, Guo Z, Gearing M, Chen G (2014) Tonic inhibition in dentate gyrus impairs long-term potentiation and memory in an Alzheimer's [corrected] disease model. Nat Commun 5:4159.

Zhang N, Peng Z, Tong X, Lindemeyer AK, Cetina Y, Huang CS, Olsen RW, Otis TS, Houser CR (2017) Decreased surface expression of the $\delta$ subunit of the GABA receptor contributes to reduced tonic inhibition in dentate granule cells in a mouse model of fragile $\mathrm{X}$ syndrome. Exp Neurol 297:168-178.

Zhong W, Cui N, Jin X, Oginsky MF, Wu Y, Zhang S, Bondy B, Johnson CM, Jiang C (2015) Methyl CpG binding protein 2 gene disruption augments tonic currents of $\gamma$-aminobutyric acid receptors in locus coeruleus neurons: impact on neuronal excitability and breathing J Biol Chem 290:18400-18411. 